\title{
Therapeutic enhancement of antibiotics using probiotics: a review
}

\author{
Bharat Kwatra ${ }^{1 *}$, Rajani Raghavendra ${ }^{2}$, \\ Rutuja Sandip Patre ${ }^{3}$, Dhvani H. Kuntawala ${ }^{4}$
}

\author{
${ }^{1}$ Invenzion Labs Inc, New Delhi, India \\ ${ }^{2}$ University of New South Wales \\ ${ }^{3}$ University of Glasgow \\ ${ }^{4}$ University of Aveiro
}

Received: 29 April 2021

Revised: 26 June 2021

Accepted: 29 June 2021

\section{*Correspondence:}

Bharat Kwatra,

Email: bkwtara999@gmail.com

Copyright: ( $)$ the author(s), publisher and licensee Medip Academy. This is an open-access article distributed under the terms of the Creative Commons Attribution Non-Commercial License, which permits unrestricted non-commercial use, distribution, and reproduction in any medium, provided the original work is properly cited.

\begin{abstract}
This article was an examination of the therapeutic enhancement of antibiotics using probiotics. The scientific development and subsequent enhancement of antibiotics continued to influence the researchers all over the globe today. This article examined the research done and published by researchers and scientists. Consideration of current trends and data in scientific queries and demonstrated further aspects of probiotics and antibiotics. Additionally, this article explored options for therapeutic enhancement of ofloxacin, amoxicillin and cefuroxime.
\end{abstract}

Keywords: Antibiotics, Probiotics, Ofloxacin, Amoxicillin, Cefuroxime, Review, Therapeutic enhancements

\section{INTRODUCTION}

Antibiotics are substances that inhibits the growth and replication of bacterium or kills it outright targeting any infection within the body. Antibiotics are used to treat bacteria, infections, some antibiotics which are of the broad spectrum also attack all bacteria including the beneficial ones. Being one the most common classes of drugs today, antibiotics are used in medicine to possibly constitute surgeries around the world. With the effectiveness and the reaction of the body, some antibiotics cause side effects such as nausea, vomiting, fever.

Probiotics are live helpful bacteria and yeast that support the digestive system. There are two important classes of probiotics, Lactobacillus and Bifidobacterium and another Saccharomyces boulardii (yeast). Both these bacteria help in digestion of lactose and ease out symptoms of irritable bowel syndrome.

A paper on probiotics, Williams study, explains how probiotics exerts their beneficial effects through various mechanism including lowering intestinal $\mathrm{pH}$, decreasing colonization and invasion by pathogenic organisms and modifying the host immune response. ${ }^{1}$

With an insight on antibiotics and probiotics, this paper aimed to explain in depth on how the use of probiotics can enhance the performance of antibiotics. The logical explanation of the theory of using probiotics with antibiotics is simple. While antibiotics kill off bad bacteria, they can also disrupt the gut's complex microbiome (the microscopic community of bacteria that work to run everything smoothy) probiotic treatment at this stage restore the good bacteria in the gut. Since the antibiotics act 
as a powerful defence against infections, it is important to restore the balance with the use of probiotics. According to the paper on probiotics as mentioned earlier, probiotics provide an excellent overall safety profile when taken at the right amount. The intestines house more than 100 trillion types of bacteria. They break down the food, help maintain a strong immune system and maintain the natural order of bodily functions. Some doctors believe that taking probiotic supplements during antibiotic treatment can reduce the negative effects on the gut flora and potentially boost immune response in the process.

Boyanova and Mitov explained probiotics used with antibiotics and how the probiotics improve the antibiotic therapy as they reduce microbial adhesion and growth by bacteriocins or other inhibitory compounds, possess immunomodulatory properties and improve intestinal barrier integrity. ${ }^{2}$ In patients treated with antibiotics, probiotics promote the recovery of commensal microbiota and increase treatment tolerability.

\section{METHODS}

The study was conducted using four databases google scholars SAGE, DOAJ and PubMed. Selection of papers were done based on keywords and theme relevant to this review. Further the published papers from these databases were arranged in systematic order with respect to year of publication.

\section{DISCUSSION}

\section{Therapeutic enhancement of ofloxacin using probiotics}

Ofloxacin a review of its antibacterial activity, pharmacokinetic properties and therapeutic use

Ofloxacin is a new generation fluorinated quinolones that is used orally as an antibacterial drug active against most gram-negative bacteria many gram-positive bacteria and some anaerobes. The clinical trial results of ofloxacin have proved to help against a wide range of infections, proven effectiveness with a high percentage of infections caused by gram-negative organisms slightly less effective against gram-positive infections and effective against some anaerobic infections. Studies by Monk et al, although well rated and tolerated, it is considered expensive, thus providing vulnerable alternative to other antibacterial drugs. ${ }^{3}$ The structure of ofloxacin is related to that of nalidixic acid. Ofloxacin is a fluorinated quinolone. Some results if clinical trials with ofloxacin have confirmed that the potential use in a wide range of infections indicated both by its antibacterial and pharmacokinetic profiles. Clinical efficacy has also shown results in systemic infections and anaerobic infections. Ofloxacin was slightly less potent an inhibitor of Providencia. Serratia and Pseudomonas species, although against Pseudomonas aeruginosa it had similar activity to enoxacin or norfloxacin was at least as potent as gentamicin and was several times more potent than aztreonam or cefotaxime.
According to this study, ofloxacin has been administered by 16,000 patients in clinical trials and the amount of side effects have been reported as $3.3 \%$ of this population. The incidence of side effects did not appear to be dose related, and serious reactions were rare. Gastrointestinal symptoms mainly nausea/vomiting, pain/discomfort, diarrhoea and anorexia were reported most frequently, followed by central nervous system events and dermatological or hypersensitivity reactions. The recommended oral dosage of ofloxacin in respiratory tract infections is 400 to $600 \mathrm{mg}$ daily and in urinary tract infections it is 200 to $600 \mathrm{mg}$ daily, in divided doses (usually twice daily) for 3 to 10 days. Higher dosages (up to $800 \mathrm{mg}$ daily) may be required in patients with severe or complicated infections. Treatments have been successful for over 3 months in patients and the tolerability for ofloxacin is monitored frequently. Ofloxacin is contraindicated in children or adolescents during the growth phase and the drug should not be administered during pregnancy or in breastfeeding mothers. Many studies in patients with respiratory or urinary tract infections demonstrated that ofloxacin was effective both in acute or chronic infections as well as in patients with or without underlying complications. In patients with skin and soft tissue infections ofloxacin was as effective as co-trimoxazole and tended to be more effective than cefaclor or doxycycline and in obstetric and gynaecological infections it was at least as effective as amoxycillin or doxycycline. Importantly, ofloxacin has a broader range of in vitro antibacterial activity than these other orally administered antibacterial drugs. Indeed the in vitro activity of ofloxacin surpasses that of many broad spectrum antibacterial drugs available only in parenteral formulation and most closely approaches that of aztreonam, although ofloxacin is active against $P$. aeruginosa. Ofloxacin has been shown to be well tolerated during both acute and long term administration. The incidence of superinfection would seem to be quite low and available evidence indicates that organisms do not readily develop resistance to ofloxacin. Therefore, ofloxacin should prove to be a valuable broad spectrum, orally administered antibacterial drug for use in a wide range of clinical infections.

\section{Antibiotic resistance of probiotic organisms and safety of probiotic dairy products}

Intrinsic resistances are common in Lactobacillus species, some of them being tetracycline, vancomycin, erythromycin, streptomycin, clindamycin, gentamicin, oxacillin and lincosamide. There are resistant markers that frequently detect the resistance strains in the Lactobacillus. Clinical cases of endocarditis, septicemia, bacteremia and septic arthritis from studies conducted by Ashraf et al due to the species of Lactobacillus, Saccharomyces, Leuconostoc, Pediococcus and Bifidobacterium have been reported in patients with some underlying medical conditions. ${ }^{4}$ The role of antibiotics has been a very significant mark in the resistance of bacteria. According to WHO the rate of emergence of antimicrobial resistance is expected to be increased by misuse of antibacterial 
substances. The European food safety authority has assessed and evaluated the antibiotic resistance determinants in lactic acid bacteria (LAB). According to this, some members of Lactococcus and Lactobillus are safe and some are pathogens. There are three types of antibiotic resistance, natural (intrinsic or innate), acquired and mutational. For example, the colonic bacteria in the colon act as reservoirs for resistance genes that can be acquired for ingested bacteria. And the reservoir hypothesis is that the commensal bacteria in the colon including those that could act as opportunistic pathogens and those that are truly non-pathogenic, exchange DNA with one another. This reserve hypothesis suggests that the antibiotic resistant bacteria came into existence because of the selective pressure applied by the antibiotic drugs. We can notice a substantial decline in the population of the bacteria after the antibiotic treatment, naturally thrive of the resistance bacteria. There are different methods to determine the antibiotic resistance of the bacteria such as Kirby Bauer (disc diffusion) method, Stokes method, Etest (based on antibiotic diffusion), agar and broth dilution or agar dilution methods for the determination of minimum inhibitory concentration (MIC). The Etest (Epsilometer Testprinzip, Ellipse gradient test AB Biodisk) is a popular quantitative technique for determining antimicrobial susceptibility. These methods have been tested and compared for different LAB and Bifidobacteria. Regarding the prevalence of antimicrobial resistance of Enterococcal strains in different environments, the frequency of it were much lower in food isolates in comparison to clinical strains. In findings by Blandino et al (2008) where $E$. faecium derived from probiotic product from Italy was susceptible to all tested antibiotics including ampicillin, cefaclor, cefotaxime, erythromycin, ciprofloxacin and gentamicin. A strain of $S$. thermophilus isolated from a probiotic product available in Italy was found resistant only to ciprofloxacin among the tested antibiotics. Selective pressure of using antibiotic in both human and animal treatment and dissemination of antibiotic resistance bacteria has the possibility to aggravate acquisition and spread of resistant genes. The probiotic organisms are considered to pool resistant genes and to transfer to the pathogenic bacteria. To avoid this possibility, MIC could be determined by most relevant antimicrobials on genetic grounds. Major of the resistance found in the species were intrinsic type. Intrinsic resistance and resistance due to mutation of chromosomal genes present a low risk of horizontal dissemination and such strains should be acceptable for food consumption. However, acquired resistance mediated by other genes could be a risk and antibiotic resistance with novel strains must be tested for dairy products. In conclusion, the selective pressure of using antibiotic in both animal and human treatment has the possibility to aggravate the spread of resistant genes.

Table 1: Therapeutic enhancement of ofloxacin using probiotics.

\begin{tabular}{|llr|}
\hline $\begin{array}{l}\text { Sr. } \\
\text { No. }\end{array}$ & Paper title & Year \\
\hline 1. & $\begin{array}{l}\text { Monk, Campoli-Richards DM. Ofloxacin a review of its antibacterial activity, pharmacokinetic } \\
\text { properties and therapeutic use. }\end{array}$ & 2013 \\
\hline 2. & $\begin{array}{l}\text { Ashraf R, Shah NP. Antibiotic resistance of probiotic organisms and safety of probiotic dairy products. } \\
\text { International Food Research Journal. 2011;18. }\end{array}$ & 2011 \\
\hline 3. & $\begin{array}{l}\text { Tripathy S, Adhya A. Ofloxacin induced leucopenia in complicated falciparum malaria: a case report. } \\
\text { Cases J. 2009;2. }\end{array}$ & 2009 \\
\hline & $\begin{array}{l}\text { Sharma JB, Chanana C, Kumar S, Roy K, Malhotra N. Comparison of ofloxacin and ornidazole with } \\
\text { probiotic versus doxycycline and metronidazole for the outpatient treatment of pelvic inflammatory } \\
\text { disease. J K Sci. 2007;9. }\end{array}$ & 2007 \\
\hline 5. & Ishibashi N, Yamazaki S. Preobiotics Safety. & 2001 \\
\hline 6. & $\begin{array}{l}\text { Hayakawa I, Atarashi S, Yokohama S, Imamura M, Sakano K, Furukawa M. Synthesis and antibacterial } \\
\text { activities of optically active ofloxacin. Antimicrob. Agents Chemother. 1986;29(1):163-4. }\end{array}$ & 1986 \\
\hline
\end{tabular}

\section{Ofloxacin induced leucopenia in complicated falciparum malaria: a case report}

This study by Tripathy et al aimed to present a case of a 44 year old female with a diagnosis of malaria, treated with intravenous ofloxacin and treated the antibacterial activity with malaria. ${ }^{4}$ Shortly after administration and resolved following discontinuation of the drug. Drug induced leucopenia (low white blood count due to an underlying disease) complicates any clinical situation. In this case study, ofloxacin induced leucopenia in a patient with falciparum malaria to which the condition reverted to normal. Shortly after stopping the drug, cases coexisting malaria and unknown areas of complicated side effects rose. A dilemma of whether or not to continue the drug if the adverse effects were caused nu the drug. Ofloxacin was administered to a 44 year old female malarial patient with symptoms of fever, diarrhoea and vomiting. Later she was diagnosed to have Plasmodium falciparum malaria elsewhere by immunochromatographic test. Prior treatment received was parenteral artesunate for three days. 
A worsening clinical status necessitated referral to our institute for management. She was resuscitated with intravenous fluids and inotropic agents and medically examined to see any further changes. Two days later, there was a renal failure and diarrhoea, further leading to anemia. At this stage stool culture grew colonies of Escherichia coli which were sensitive to ofloxacin and carbapenem. The patient was started on intravenous ofloxacin (200 mg twice a day) after adjusting for impaired renal function. After five days of treatment patient continued to deteriorate with severe leucopenia. No infections were picked up from the $\mathrm{X}$-ray done and ultrasound cleared as normal. Shortly after the administration lucopenia was developed and upon the discontinuation of the drug ofloxacin use, white blood cell count returned to normal. The stability was seen only after seven days and normal levels of cell count were only after day ten. Hence ofloxacin is suspected to cause leucopenia. Ofloxacin is a commonly used fluoroquinolone agent in gastrointestinal infections and been associated with adverse effects such as seizures, hypersensitivity vasculitis, hemoglobinurea. Drug induced leucopenia was the provisional diagnosis. The patient was on injection ofloxacin, artesunate, prebiotics and probiotics for diarrhoea, rabeprazole, salbutamol and dopamine infusion. Ofloxacin was discontinued at this stage. Hematological profile showed signs of stabilization on day seven. The cell count reached near to normal. Bone marrow counts revealed an increase in levels of promyelocyte and myelocyte. Leucopenia in a critically ill patient can be attributed to various causes. The Falciparum malaria is associated to anemia and thrombocytopenia. Other infections can cause damage to the connective tissue and can cause activation syndrome and leucopenia. Druginduced leukopenia can occur in a dose-dependent relationship or hypersensitivity reactions. Although leucopoenia is an adverse effect of several drugs. It was ofloxacin related in the malaria patient. Healthcare workers should be wary of treating patients with ofloxacin. Leucopoenia is a well-recognized adverse effect of several drugs. We reported a case of ofloxacin-associated leucopoenia during treatment of a patient with malaria. Healthcare personnel should be aware of this possible adverse reaction in patients treated with ofloxacin. A high degree of suspicion assumes special importance in this subgroup of critically ill patients with malaria where hematologic aberrations are common.

Comparison of ofloxacin and ornidazole with probiotic versus doxycycline metronidazole for the outpatient treatment of pelvic inflammatory disease

This study by Sharma aimed to compare the efficacy, cost and effectiveness of ofloxacin, ornidazole, serratiopeptidase and Saccharomyces Boulardii combination with traditional doxycycline and metronidazole combination with serratiopeptidase in the outpatient management of pelvic inflammatory disease. ${ }^{4}$ Hundred and ninety three women suffering from pelvic inflammatory disease (PID) were subjects to this experiment. Clinical examinations were held and different dozes of treatments were given and follow up for every 2 days. After 2 weeks of relief and side effects, conclusions were drawn to compare the efficacy of the drugs. PID is a polymicrobial infection of upper genital tract. It is a serious condition that is caused in sexually active women with infertility and ectopic pregnancies (a pregnancy in which the fertilised egg implants outside the uterus). This is most commonly caused by aerobic and anerobic pathogens and are treated with genital tract pathogens via drugs. The most common ones are doxycycline and metronidazole, although has side effects in the gastrointestine. Whereas ofloxacin alone or along with ornidazole have recently been used with good results. This papers discussed the efficacy of ofloxacin, ornidazole with probiotic $S$. Boulardii and Lactic acid bacillus (to decrease GI side effects) with the traditional doxycycline and metronidazole as outpatient syndromic management of PID. This paper demonstrates the study of 193 women suffering with PID symptoms like vaginal discharge, pruritus vulvae with or without vaginal pain and lower abdominal pain over a period of 6 months. Detailed tests were conducted to observe the type of discharge, consistency, colour and smell, the area around the vagina and the cervix and any other abnormalities. The women were divided into different groups of what would be tested. Group 1, total of 93 women were tested with combination treatment of ofloxacin (400 mg)/ornidazole (500 mg)/S. Boulardii (2 million spores)/Lactic acid bacillus $(60$ million spores)/Serratiopeptidase $(10 \mathrm{mg}$ ) once a day for a total of 10 days. Group 2 consisted of 95 women who received treatment with standard doxycycline (100 mg twice daily) with metronidazole ( $400 \mathrm{mg}$ three times a day) along with $10 \mathrm{mg}$ of serratiopeptidase once daily for 10 days. Follow up was done for every 2 days and symptomatic relief and improvement were being observed. The results showed no significant differences in the symptoms among the group of women. However, the side effects were less common in group 1 who used ofloxacin. The only mentionable difference with the inclusion of ofloxacin were the increase of price by $\$ 3.00$ USD. In the study, we compared the efficacy of ofloxacin $(400 \mathrm{mg})$ with ornidazole $(500 \mathrm{mg})$ with Saccharomyces once a day for 10 days with a 10 day course of doxycycline (100 mg twice daily) and metronidazole (400 mg thrice a day). To conclude, syndromic approach using a combination of ofloxacin (400 $\mathrm{mg}$ ) with ornidazole $(500 \mathrm{mg})$ appears to be an efficient and cost effective modality for syndromic management of PID.

The syndromic approach to identify the efficacy of the drugs based on the symptom and sign of PID depending on knowledge of the most common causative organism of these syndromes and their antimicrobial susceptibility. Syndromic approach has its own advantages of expedited care, cutting cost of laboratory experiments, better patient compliance and lesser follow up before starting the treatment. The only disadvantage of this approach is that there could be cost of over diagnosis for developing antibiotic resistance. 


\section{Probiotics and safety}

Traditionally, bacterial species are used in probiotics are considered as safe. These mainly include the Lactic acid bacteria and the Bifidobacteria that stay in the intestinal tracks of humans and animals. Thus, questioning the safety of probiotics from the infections perspective. The article by Ishibashi et al describes the safety of probiotic bacteria by isolating it from the infection and reviewing each factor such as pathogenicity, infectivity, toxicity and intrinsic properties of the bacteria. ${ }^{6}$

Probiotics are defined as food or in other terms drug that contain microbes which provides beneficial physiologic effects through microbial actions when ingested. The microbes used in the probiotics must be able to perform the activities needed in the host's body. For example, the human digestive track contains lot of microbes and thus the balance of these microbial activities influences the functioning of the intestine and the environment around it. Among the numerous microbial activities the ones that prove to be the most beneficial are the ones that are selected as probiotics. Often, most probiotics are sold as drugs or added in food. Some of them that have extensively been used in food processing are Lactobacillus, Leuconostoc, and Pediococcus, thus it's important to ensure that these probiotics are safe and the ingestion of foods containing live bacteria, dead bacteria and metabolites of these microorganisms has taken place for a long time. Lactic acid bacteria and Bifidobacteria isolated from endocarditis, bacteria and bloodstream and local infections. Most of the bacterial species that constitute probiotics have actually been isolated from infection sites, verification of the safety of probiotics used industrially and commercially is important. One of the requisite of probiotic safety is the absence of pathogenicity and infectivity. The isolation of Lactic acid bacteria and Bifidobacteria enough though has no infectivity are frequently isolated form infections, which are generally due to the result of opportunistic infectivity. The causes of opportunistic infection include skin injury, chronic diseases, cancer and drug-induced abnormality. The Lactic acid bacteria as well as the Bifidobacteria could invade the host's body though bacterial translocation and other means resulting in bacteremia due to infections. To access such a situation is difficult and the invasion of the bacteria cannot be tracked. The infection doesn't occur often in health animals even if the bacteria is administered orally, this happens only when the bacteria is weakly infective. Same with a strongly infective bacteria, it is impossible to establish the infection using single species, various methods are required to find out the infection.

We can see the invasion route of the bacteria via translocation which eventually causes bacteremia, further progressing into organ failure. Bacterial translocation is caused due to the diminished intestinal barrier mainly due to the passage of bacteria across the mucous membrane. It then moves to the lymph nodes and other organs causing bacteremia, which further progresses as multiple organ failure and septicaemia. The method to induce translocation is via the treatment of antibiotics, nuosuprressive agents or a combination of both. Another method is the germ-free mice as bacterial translocation occurs for a long period of time. This method is caused by the immature intestinal barrier and under developed immunity of the lymphatic system in germ-free animals. Berg and Garlington observed translocation in E. coli or $L$. acidophilus-monoassociated gnotobiotic mice and found translocation to the MLN, spleen and liver over a long duration. It is not easy to assess the safety of probiotics by properties and functionality of the bacteria. Although, in vitro tests could be a close way to analyse the path of the bacteria. Another requisite of probiotics is that the probiotic bacteria should not produce harmful substances by metabolic activities. A method of testing this theory is to determine if the bacteria coverts good components into secondary harmful substances to the host. Another test for the assessment of safety is the platelet aggregating activity which contributes to the progression of the infective endocarditis.

The ability to cause the infection by the bacteria seems to be a difficult task, but the level of invasion is identifiable. The platelet aggregation is another factor that reveals the invasion by the bacteria due to the high molecular weight. The relation of this phenomenon with infections including endocarditis remains unknown through this process. Another relation that requires further studies is that between the genetic characteristics of the bacteria and the type of infection.

Synthesis and antibacterial activities of optically active ofloxacin

Ofloxacin is an isomer that belongs to the methyl group at $\mathrm{C}-3$ position in the oxazine ring resulting in an asymmetric centre. This paper described the relationship between the optical isomers and the antibacterial potencies of ofloxacin. Ofloxacin is a nalidixic acid and some of these category acids have found to have a potent activity against gramnegative bacteria, which is essential for the antibacterial activity. Some new nalidixic acid have found to have antibacterial activities not only against gram-negative bacteria but also gram-positive bacteria, one of them being ofloxacin from findings in Hayakawa. ${ }^{5}$ Ofloxacin is a tricyclic structure, with a methyl group at the $\mathrm{C}-3$ position in the oxazine ring, resulting in an asymmetric center, resulting in (-)-ofloxacin and (+)-ofloxacin. The synthesis of optically active ofloxacin + and - through optical resolution of racemic intermediate ofloxacin by high pressure liquid chromatography. The result of separation of ofloxacin at high pressure indicated that the configuration of the methyl group at the $\mathrm{C}-3$ position of ofloxacin has significant effects on the antibacterial activity. Ofloxacin is very potent member of fluoroquinolone family with broad spectrum against a wide variety of bacterial infections; its structure. Literature reveals that there are problems in its radiolabelling with such as use heating and instability in serum, thus there is a need to search more efficient, robust 
method for the development of ofloxacin kits, its radiolabelling and in vivo studies to sketch the whole profile of the pharmacokinetics and efficacy. Two optically active (100\% enantiomeric excess) isomers of ofloxacin multiplication of gram-positive and gram-negative bacteria than the other, (+)-ofloxacin and approximately two times more active than the race mate, (+)-ofloxacin. The result of the experiment conducted was that $\mathrm{C}-3$ position of ofloxacin has effects on antibacterial activity. Hence, the stereochemistry at this position plays an important role to inhibit DNA gyrase. This study was to establish a relationship between two optical Isomers of Ofloxacin and their antibacterial potencies. Numerous substitutes for the above were also synthesized, all of them DO NOT have an asymmetric center. The compound ofloxacin has antibacterial properties.

\section{Therapeutic enhancement of amoxicillin using probiotics}

\section{A relative study of effect of probiotic with amoxicillin on Danish and Portuguese populations}

Use of antibiotics is often associated with AAD (antibiotic associated diarrhea). To overcome this problem probiotics treatment with antibiotic seemed successful. Previous study suggests lower risk of AAD, when probiotics were taken with antibiotics. To evaluate the effect of probiotics along with antibiotics, a study on the samples from
$( \pm)$-ofloxacin were prepared by use of their optically resolved synthetic intermediates. One of the isomers, (-)ofloxacin, was 8 to 128 times more potent in inhibiting the

Denmark and Portugal population was conducted. Target population were the patients that were prescribed antibiotics regardless of a simultaneous intake of probiotics. Amoxicillin-clavulanic acid was used as antibiotic in the study. The result suggested no diarrhea in $91 \%$ of patients who took probiotics. $16.66 \%$ of the sample population without probiotic showed higher expression of AAD during and after treatment, while only $12.5 \%$ with probiotic population showed diarrhea during but no diarrhea after treatment. ${ }^{9}$ Overall, the study indicated that the use of probiotic along with amoxicillin-clavulanic acid or any other antibiotic prevents AAD irrespective of the age group. Patients in both populations were surveyed. The occurrence of diarrhea was studied post-treatment. Patients that suffered from diarrhea by the time the treatment started were excluded from study. The results suggested that diarrhea was more prevalent in the population that did not take probiotic than the one that took probiotic along with amoxicillin-clavulanic acid. $91 \%$ of patients that took probiotics showed no diarrhea, while the group without probiotic intake showed $16.6 \%$ occurrence of AAD during treatment $(16.6 \%, \mathrm{~N}=27)$ and after treatment. In general, the use of probiotic with amoxicillin-clavulanic acid suggested decline in the occurrence of diarrhea in both populations (Stillits et al 2010).

Table 2: Therapeutic enhancement of amoxicillin using probiotics.

\begin{tabular}{|llc|}
\hline Sr. No. & Paper Title & Year \\
\hline 1. & A relative study of effect of probiotic with amoxicillin on Danish and Portuguese populations. & 2010 \\
\hline 2. & $\begin{array}{l}\text { A comparative study of evaluation of Helicobacter Pyroli eradication in pediatric patients by } \\
\text { triple therapy with lactoferrin and probiotics and triple therapy alone. }\end{array}$ & 2012 \\
\hline 3. & Effect of adjuvant probiotics on triple therapy for Helicobacter pyroli infection. & 2012 \\
\hline 4. & Effects of probiotics on microbiota interference after antibiotic treatment. & 2012 \\
\hline 5. & $\begin{array}{l}\text { Effect of galacto-oligosaccharides on intestinal microbiota of healthy adults undergoing } \\
\text { amoxicillin treatment. }\end{array}$ & 2014 \\
\hline 6. & $\begin{array}{l}\text { Effect of probiotics on efficacy and tolerability of triple therapy in eliminating Helicobacter } \\
\text { pyroli: A meta-analysis. }\end{array}$ & 2015 \\
\hline 7. & $\begin{array}{l}\text { An experimental study to evaluate effect of probiotic plus prebiotic on efficacy and tolerance of } \\
\text { Helicobacter pyroli } \text { eradication Quadruple therapy. }\end{array}$ & 2016 \\
\hline 8. & $\begin{array}{l}\text { Design and testing of an oral system for double delivery of amoxicillin and Lactobacillus } \\
\text { acidophilus. }\end{array}$ & 2016 \\
\hline 9. & $\begin{array}{l}\text { Randomized controlled study to evaluate effect of Saccharomyces boulardii CNCMI-745, } \\
\text { amoxicillin-clavulanate and combined effects of both on healthy gut microbiota. }\end{array}$ & 2017 \\
\hline 10. & Lactobacillus Shirota improves efficacy of amoxicillin sulbactam. \\
\hline
\end{tabular}

Table 3: Occurrence of side effects in both groups.

\begin{tabular}{|llll|}
\hline Side effects & $\mathbf{A}(\mathbf{N}=\mathbf{3 4})(\boldsymbol{\%})$ & $\mathbf{B}(\mathbf{N}=\mathbf{3 4})(\boldsymbol{\%})$ & P value \\
\hline Epigastric pain & $6(17.6)$ & $2(5.8)$ & $<0.05$ \\
\hline Nausea & $3(8.8)$ & $1(2.9)$ & $<0.05$ \\
\hline Vomiting & $2(5.8)$ & 0 & $<0.05$ \\
\hline Diarrhea & $8(23.5)$ & 0 & $<0.05$ \\
\hline Constipation & $2(5.8)$ & $25.8)$ & NA \\
\hline
\end{tabular}


A comparative study of evaluation of Helicobacter Pylori eradication in pediatric patients by triple therapy with lactoferrin and probiotics and triple therapy alone

H. pylori infection causes gastritis, peptic ulcers, gastric cancer and other severe diseases. A standard treatment for H. pylori is the 7-14 days of triple therapy. However, due to various factors eradication rates by triple therapy have been reduced. To overcome this, adjuvant therapies are now considered such as with probiotics. This study was conducted to evaluate probiotic effect on $H$. pylori eradication rates in children. $H$. pylori diagnosed patients where in 2 therapy groups (A and B). Both groups received triple therapy. B also received probiotic (probinulcadigroups). After completion of the study, 26 patients (76.3\%) in group A and 30 patients $(88.2 \%)$ in group B showed H. pylori eradication. Probiotic supplementation significantly reduced the side effects of treatment. ${ }^{10}$

Patients diagnosed of $H$. pylori from July 2008 to July 2011 were considered for study. Group A were administered omeprazole ( $1 \mathrm{mg} / \mathrm{kg}$ before breakfast), amoxicillin (50 $\mathrm{mg} / \mathrm{kg}$ after meals) for 7 days, while $\mathrm{B}$ with same antibiotics and probiotic once a day for 7 days. The probiotic ( $5 \mathrm{~g} /$ day dose) probinul-cadigroup was used for study because of higher concentration of a range of bacteria as well as inulin as prebiotic. Negative C13UBT result 4 weeks after therapy indicated successful eradication. $68 \mathrm{H}$. pyroli infected children (32 males and 36 females) were considered for study.

Nausea, vomiting, diarrhea and epigastric pain was more prevalent in group A $(\mathrm{R}<0.05)$ than in group $\mathrm{B}$ as seen in Table 3. 26 patients $(76.4 \%)$ in group A and 30 patients (88.2\%) in group B suggested $H$. pylori eradication with relatively greater success rate for eradication in group B than A. H. pylori infection is more prevalent in childhood and is hypothesized to be transferred from mother to child. Triple therapy eradication rates have been declining and these treatments result in poor compliance leading to side effects prominently in children. To reduce the side effects of these treatments and increase eradication rates adjuvant therapies are now considered. Most adjuvant therapy trials used probiotics as observed in previous studies. Combined treatment with (triple therapy+probiotic+bovinelactoferrin) was more successful than triple therapy alone. Probinul probiotic supplementation to standard triple therapy greatly reduced side effects $(61.5 \%$ in group A; $14.5 \%$ in group B). The study also had certain shortcomings as no placebo control group was considered and the sample size for study was small. In general, probiotic along with triple therapy greatly reduced the occurrence of various side effects and eradication rates were slightly higher in group A thus, indicating that probiotics with triple therapy can be used to increase eradication rates in $H$. pylori infected children (Tolone et al 2012).
Effect of adjuvant probiotics on triple therapy for $H$. pylori infection

H. pylori causes fatigue, diarrhea, peptic ulcers, nausea. Eradicating $H$. pylori from the body is difficult due to antibiotic resistance, therefore a combination of 3 antibiotics known as triple therapy is often given. However, even the triple therapy showed decline in its effectiveness due to antibiotic resistance. To investigate probiotic effect in pylori infected patients, pre and post probiotic administration before and after triple therapy respectively was examined. Since $L$. acidophilus has been effective in treating pylori, it was used as the major probiotic. RUT (rapid urease test) and C13 and C14 urea breath test (UBT) suggested that both pre and post probiotic treatment improved eradication rates with post probiotic group indicating more eradication.

Patients aged 18-65 diagnosed with $H$. pylori were considered for the study and enrolled patients in $1: 1: 1$ into 3 groups. OCA (triple therapy) group received standard triple therapy of $(20 \mathrm{mg}$ omeprazole, $500 \mathrm{mg}$ clarithromycin, $1000 \mathrm{mg}$ amoxicillin) for 7 days. POCA (probiotic pretreated group) received 2 weeks of probiotics containing $3 \times 10^{\wedge} 7 \mathrm{l}$. acidophilus per day before 1 week of triple therapy and the OCAP group (probiotic post treated group) was given 1 week of triple therapy followed by 2 weeks of probiotics. Randomization was done by SPSS 18.0 software.

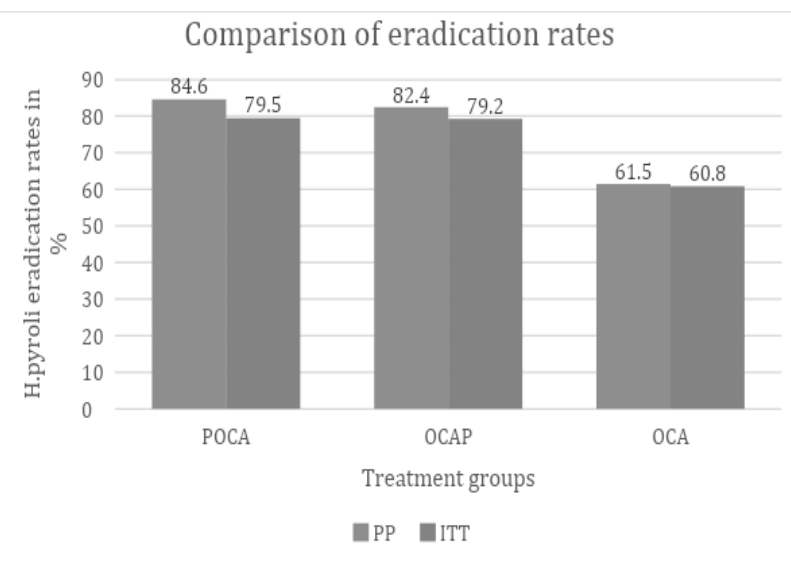

Figure 1: $H$. pylori eradication rates in the treatment groups of triple therapy, triple therapy with and without probiotics; POCA: probiotic pretreated group, OCAP: probiotic post treated group, OCA: standard therapy group, PP: per protocol, ITT: intention to treat.

After triple therapy, C13 or C14 UBT test was negative in $171 / 228$ patients $(75 \%)$. Per protocol (pp) investigation suggested higher eradication rates in the POCA and OCAP groups then in OCA. IIT (intention to treat population showed greater eradication rates in the POCA and OCAP group. Study suggested use of probiotics pre and post triple therapy improved eradication rates of $H$. pylori infection. Although post probiotic treatment after triple therapy 
greatly improved eradication rate. ${ }^{11}$ A study revealed pretreatment with L. acidophilus, S. faecalis and B. subtilis 2 weeks before triple therapy increases eradication rate. Results of this study are consistent with previous studies. However, the optimal dose and the period of treatment is yet to be determined. Post probiotic treatment after triple therapy suggested higher eradication rates than triple therapy alone (from $60.8 \%$ to $79.2 \%$ ). The study also reveals that $H$. pylori might be less sensitive to single strain than multi-strain probiotics. To sum-up, pre and post probiotic treatment improved $H$. pylori eradication of triple therapy (Du et al 2012).

Effects of probiotics on microbiota interference after antibiotic treatment

Antibiotics are often associated with certain side effects, where they affect the activity of intestinal microbiota. This interference in the regular microbiota leads to AAD (antibiotic associated diarrhea). Thus, probiotics act as a good stabilizer for the intestinal microbiota. To understand their effect, a random double blind group study with 2 species of probiotic L. acidophilus and Bifidobacterium lactis was done during and after antibiotic treatment with placebo group as control. Realtime PCR was used to investigate if the probiotic helped restore microbiota and its effect on $\beta$-lactam resistance. Probiotic treatment group showed higher faecal counts of L. acidophilus and B. lactis after 8th day and 15th day compared to placebo group. Both groups indicated higher $\beta$-lactam resistance after antibiotic exposure.
The study divided participants by gender at a ratio of $1: 1$ and all volunteers received amoxicillin $(875 \mathrm{mg})$ and clavulanate $(125 \mathrm{mg})$ from day 1 to day 7 and then randomly allocated to receive probiotic and placebo daily from 1 to 14 . Study products were: $12.5 \times 10^{\wedge} 9 \mathrm{CFU} / \mathrm{d} L$. acidophilus and $12.5 \times 10^{\wedge} 9 \mathrm{CFU} / \mathrm{d} \quad B$. animalis in Hypromellose capsule with maltodextrin as excipient. The placebo group had the same capsule with only maltodextrin. Probiotic group showed increased faecal counts of L. acidophilus at the end of antibiotic treatment compared to the placebo group.

Probiotic group also indicated more levels of $B$. lactis and L. acidophilus compared to placebo. Post antibiotic treatment, 16 participants in the probiotic group and 14 from placebo suggested $\beta$-lactam resistance $(p=0.924)$. GSRS scores were low. Both groups indicated higher Bristol scale values with highest on 3rd day of antibiotic treatment. $^{12}$ This study involved healthy adults as volunteers that were given same dose of antibiotic for same period, which established a baseline and eliminated any possibilities of variations. High levels of L. acidophilus ATCC700396 and B. lactis in probiotic group were due to consumption of these 3 strains. As observed from the results, relatively less disturbance in faecal microbiota relates with less gastrointestinal complaints by patients. Overall probiotic use led to relatively small increase in faecal levels of consumed species (influence of probiotic mix, no date).

Table 4: Number of total bacteria and Bifidobacteria spp prior treatment (mean values of log 10 copies/g faeces with SD errors).

\begin{tabular}{|llllllllllll|}
\hline Treatment (prior) & GOS & \multicolumn{1}{c|}{ Placebo (MD) } \\
\hline Subjects & 1 & 5 & 6 & 8 & 12 & 2 & 3 & 7 & 9 & 11 & SEM \\
\hline Total bacteria & 10.4 & 10.1 & 10.2 & 10.5 & 9.5 & 10.4 & 10.4 & 10.4 & 10.4 & 10.4 & 0.1 \\
\hline Bifidobacteria & 9.8 & 9.4 & 10.1 & 9.7 & 9.5 & 10.3 & 9.6 & 7.5 & 10.0 & 9.4 & 0.3 \\
\hline
\end{tabular}

\section{Effect of galacto-oligosaccharides on the intestinal flora of healthy targets undergoing amoxicillin treatment}

It has been found that most antibiotics are associated with various side effects causing intestinal disturbance. To overcome these, use of probiotics and prebiotics are considered since previous studies showed promising result of their usage. In this study 12 healthy adults were given galacto-oligosaccharides (GOS) receiving amoxicillin to study the effect of GOS on intestinal microbiota. Groups were randomly divided into GOS and placebo both receiving $375 \mathrm{mg}$ amoxicillin thrice a day. Results suggested the GOS positively affected bifidobacterial levels relatively but not significantly.

Moreover, metabolic activity of Bifidobacteria and other microbiota was more in the GOS group than the placebo group. The results were consistent with previous invitro findings where GOS positively affected recovery of beneficial bacteria after amoxicillin treatment. The parallel intervention study consisted of $12 \mathrm{~d}$ of intervention and 14 $\mathrm{d}$ of follow up. One group was given amoxicillin (AMX) and GOS and other AMX and placebo.

\section{Microbiota analysis}

Composition of microbiota was studied using intestinal (I) chip and by quantification of total and Bifidobacterium spp.

During 1st phase (day 2 and 5), number of Bifidobacterial species declined in both groups with time due to amoxicillin treatment. While, in phase 2 (day 8 and 12), no rise in GOS group was seen compared to placebo. In phase 3 (follow up day 19 and 26), number of Bifidobacteria were similar to initial numbers in both groups. 


\section{Analysis of metabolic activity of microbiota}

The metabolic activity was evaluated by determining SCFA (short chain fatty acids), monosaccharides, oligosaccharides and organic acid levels in the faecal samples. In phase 1 succinate levels rose on day 2 in GOS group (20 umol/g) and rose in placebo group on day 5 (13 umol/g faeces). During follow up period SCFA and OA levels were similar to initial values in both groups. Monosaccharides and oligosaccharides levels increased in the GOS group on day two compared to day zero by $30 \%$. The number of Bifidobacteria in the microbiota was observed more than the findings in the previous literature which was most likely due to the poor primer response. Moreover, GOS did not affect microbiota composition significantly during amoxicillin treatment but seemed to restrict reduction in bifidobacterial abundance. Recovery of microbiota composition and its metabolic activity was observed to be quicker in GOS group than the placebo group after discontinuing amoxicillin treatment rather than during.${ }^{13}$ In conclusion the in vivo study thus suggested that GOS stimulates Bifidobacteria growth post discontinuation of amoxicillin treatment and that GOS positively affects microbiota and metabolic activity recovery after discontinuation of amoxicillin treatment rather than during (Ladirat et al 2014).

Effect of probiotics on efficacy and tolerability of triple therapy in eliminating H. pylori: a meta-analysis

H. pylori affects $50 \%$ of the world population and links not only to digestion related diseases but also affects other organs leading to allergies, neurological diseases, diabetes. The standard triple therapy has showed decline in eradication rates due to antibiotic resistance. Previous studies indicated that probiotics inhibited $H$. pylori. Therefore, study was conducted to evaluate probiotics effect on efficacy and tolerability of triple therapy to eliminate $H$. pylori using a meta-analysis approach, where articles since 31 August 2014 were searched on PubMed, Web of Science and Cochrane Collaboration. 23 articles were considered for the meta-analysis. The study revealed that probiotics improved the efficacy and tolerability of triple therapy.

23 articles were shortlisted for analysis after a literature search based on the exclusion and inclusion criteria. The result in triple therapy alone showed eradication rate was 72.26\% (1464/2026; 95\% CI, 67.66\%-74.13\%) compared to a rate of $80.74 \%(1513 / 1874 ; 95 \%$ CI, $74.68 \%-82.76 \%)$ for probiotic plus triple therapy. ${ }^{14}$ Probiotic treatment along with triple therapy has showed promising results as per the meta-analysis and previous studies. Various antibiotics such as Lactobacillus gasseri OLL2716 (LG21), B. subtilis and S. boulardii can cause H. pylori elimination using different mechanism. In conclusion, meta-analysis suggested improvement of $H$. pylori eradication by triple therapy with probiotic supplementation with eradication rates $(\mathrm{OR}=0.58 ; 95 \% \mathrm{CI}$, 0.50-0.68) (Li et al 2015).
An experimental study to evaluate effect of probiotic plus prebiotic on efficacy and tolerance of $H$. pylori eradication quadruple therapy

H. pylori causes various gastrointestinal disorders. The most successful treatments have shown decline in eradicating bacteria such as triple therapy. Quadruple therapy is often prescribed in places where there is resistance to clarithromycin. However, recent findings suggested that probiotic supplementation with or without prebiotic may improve eradication rate and reduce side effects. The study focused on investigating probiotic effect with prebiotic on $H$. pylori quadruple therapy eradication rates and side effects. Seventy six H. pylori patients were randomized to undergo a quadruple therapy (bismuth, clarithromycin, amoxicillin and one placebo control). UBT was done 8 weeks after treatment. Synbiotic group suggested increased eradication rate by ITT analysis.

76 patients diagnosed with $H$. pylori were enrolled from May 2011 to March 2012. Patients that were given 14 days of quadruple therapy (consisted of bismuth sub-citrate 4 times a day, amoxicillin $1 \mathrm{~g}$, clarithromycin $500 \mathrm{mg}$ and omeprazole $20 \mathrm{mg}$ all twice a day) and were randomly divided to receive symbiotic or placebo. Patients were asked to begin symbiotic or placebo 3 days prior main treatment for better colonization of probiotic. Overall, eradication rates in total patients after treatment was $95.2 \%$ (CI 95\% 89.9-100). 79\% of these patients had non-ulcerdyspepsia (NUD). ITT analysis suggested eradication rates of $92.1 \%$ (CI 95\%; 83.5-100\%) and 63.2\% (CI 95\%; 47.9$78.5 \%)$ in synbiotic and placebo group. ${ }^{15}$

H. pylori related disorders have increased with decreased eradication rates of triple therapy and treatment resistance and failure. Probiotics are live bacteria that are beneficial for gut microbiota, while prebiotics are non-digestible food ingredients that benefit by stimulating activity of one or few bacteria in colon and synbiotics are combination of prebiotics and probiotics. This study suggested addition of synbiotic to anti $H$. pylori regimen led to increased drug tolerance as observed by ITT analysis. The study also indicated improvement of anorexia due to synbiotics. Moreover, it suggests that probiotics with prebiotics can improve eradication rate significantly (Shafaghi et al 2016).

Design and testing of an oral system for double delivery of amoxicillin and L. acidophilus

Research has been done to maintain human gut and improve human health using beneficial bacteria such as probiotics. Moreover, they are also used as adjuvant with antibiotic treatment. However, antibiotics are associated with certain side effects causing AAD. Therefore, probiotics along with antibiotics are used as treatment approach but this has certain disadvantages like when taken simultaneously the antibiotic can destroy the probiotic even before they employ their health benefits. Thus, a delayed release system for dual delivery of amoxicillin and 
L. acidophilus was designed and examined. Denatured ovalbumin matrix was designed using Box-Behnkn method before encapsulating with glycerol monostearate. Amoxicillin with encapsulated ovalbumin was incorporated into gastro-resistant capsule. In vitro characterization and analysis suggested that $L$. acidophilus probiotic was not killed by bactericidal effects of amoxicillin in the dual delivery system. ${ }^{16}$

The study was successful in designing a stable dual-release system for simultaneous delivery of amoxicillin and $L$. acidophilus. This system thus has beneficial health effects on patients undertaking antibiotic treatment wherein the beneficial gut microbiota that is destroyed is now supplemented with beneficial bacteria again. Thus, the results indicated that ovalbumin system with GMS for delayed release protected probiotic against bactericidal effects of amoxicillin. In conclusion, dual delivery system for simultaneous delivery of probiotics with amoxicillin was created and was successful in eliminating other problems such as bactericidal effect on probiotics, antibiotic associated microbiota depletion and patient noncompliance (Govender et al 2016).

Randomized controlled study to evaluate the outcome of $S$. boulardii CNCMI-745, amoxicillin-clavulanate and combined treatment of both on healthy gut microbiota

Probiotics are associated with health benefits such as maintaining intestinal microbiota and helping in digestion, while antibiotics leads to antibiotic induced gut imbalance. Thus, this study focused on investigating effect of $S$. boulardii CNCMI-745 (SB) probiotic, amoxicillinclavulanate (AC) and combination of both on the microbiota of healthy human targets. Targets were randomized into 4 groups (SB for 14 days, 7 days $\mathrm{AC}$, $\mathrm{SB}+\mathrm{AC}$, control, no treatment). Faecal samples were collected for evaluating gastrointestinal discomfort and effect on microbiota was analyzed by $16 \mathrm{~S}$ rRNA gene pyrosequencing. $\mathrm{SB}+\mathrm{AC}$ group showed less adverse events compared to AC group. AC induced increase in E. coli, Parabacteroides and Enterobacter whereas combined treatment of SB + AC declined the overgrowth of $E$. coli and also reduced values for AAD were observed. ${ }^{17} S$. boulardii reduced antibiotic induced microbiota changes and AAD as well.

The study findings were consistent with the previous literature. As seen from the results, microbiota changes were evident with antibiotic use. S. boulardii alone did not have a significant effect on the microbiota. However, addition of SB with AC affected antibiotic induced microbiota change positively. Overall, co-administration $(\mathrm{AC}+\mathrm{SB})$ led to decline in AAD without worsening other gastrointestinal disorders. Moreover, indigestion observed in the 1st week in SB group was less prominent when SB with AC was administered. However, the study had few limitations as the target population was healthy and not infected, thus the result cannot be generalized plus it was not a blinded study. Further study on infected populations should also be done to see if the results can be generalized (Kabbani et al 2017).

\section{Shirota improves efficacy of amoxicillin sulbactam}

Pneumonia is a disease caused by bacterial or any other infection which majorly affects the lungs. Children lungs are more prone to it than the adult and thus childhood fast breathing pneumonia is one of the causes of increasing children mortality. Antibiotic such as aminopenicillin $\beta$ lactamase inhibitors are used for the treatment, but these are expensive for a normal population. A study revealed using probiotics had beneficial effects in treating pneumonia. Therefore, a clinical study to evaluate the efficacy of L. Shirota with amoxicillin-sulbactam was compared with amoxicillin-sulbactam plus placebo group. 454 children (2-48 months old) diagnosed of fast breathing pneumonia were divided into 2 treatment groups amoxicillin-sulbactam plus L. Shirota (AS+LcS) and amoxicillin-sulbactam plus placebo (AS+placebo). Treatment failure till day 3 was recorded as the main outcome with treatment failure till day 12 as the secondary outcome. Also, blood samples showed lower TNF- $\alpha$ and IFN- $\gamma$ levels in serum of (AS+LcS) group compared to (AS+placebo group). 64 out of 518 patients were excluded from the study based on the exclusion criteria. AS+LcS group was given $100 \mathrm{mg} \mathrm{kg} /$ day dosage of amoxicillinsulbactam (upto $3 \mathrm{~g} /$ day) every $8 \mathrm{~h}$ along with skimmed milk containing $6 \times 10^{\wedge} 9 \mathrm{CFU}$ of $\mathrm{LcS}$. AS+placebo group was given the same dosage of amoxicillin-sulbactam for same period with skimmed milk as placebo. Blood samples of all patients were collected with $0.1 \%$ EDTA after day 3, centrifuged and stored at $-80^{\circ} \mathrm{C}$ to examine the interferon$\gamma$ and TNF- $\alpha$ levels in serum. Serum concentrations showed decrease in both IFN- $\gamma$ and TFN $\alpha$ levels in AS+LcS group than AS+placebo group. On day 6 and 12, the number of patients that suffered with pneumonia were less in $\mathrm{AS}+\mathrm{LcS}$ group as compared to $\mathrm{AS}+$ placebo. ${ }^{18}$

The results indicate that combination of L. shirota with AS improved the results of treatment as observed by highly reduced treatment failures in (AS+LcS) group as compared to (AS+placebo) group upto 12 days. Additionally, TNF- $\alpha$ and IFN $\gamma$ levels in serum declined in AS+LcS as compared to AS+placebo suggesting less inflammation. Also, $L$. shirota has evidence of safe consumption with numerous health benefits and its immune modulating properties thus improving the efficiency of amoxicillin sulbactam in treatment. In conclusion L. shirota can be used as an adjuvant for further treatment (Li et al 2018).

\section{Therapeutic enhancement of cefuroxime using probiotics}

Investigation of cefuroxime, cefotaxime and cefpirome with B. clausii biodegradation pathways

Bioremediation options are available as cephalosporin residues in the environment are of concern. B. clausii $\mathrm{T}$ when mixed with cefuroxime (CFX), cefpirome (CPR) and cefotaxime (CTX) reached a removal rate of $100 \%$ in 8 
hours. A higher removal efficiency was displayed by the co-culture of B. clausii $\mathrm{T}$ and $B$. clausii $\mathrm{O} / \mathrm{C}$ for the mixture of CFX, CPR and CTX than the pure culture of B. clausii $\mathrm{O} / \mathrm{C}$. B. clausii $\mathrm{T}$ by alleviation of the biotoxicity of CFX and CPR. B. clausii T and B. clausii O/C had a lower biotoxicity of for CFX and CPR change products removed than the pure cultures. Real time PCR was used to notice the variations in the expression levels of the antibiotic resistance genes of $B$. clausii during the degradation of CFX and CPR. The expression of the $\beta$-lactamase gene bcl1 was enhanced by CFX and CPR. Decarboxylation, hydrolysis and deacetylation are the main mechanism of CTX biodegradation by B. clausii. Removal efficiency of different combinations of CFX, CTX and CPR by pureand co-cultures of B. clausii $\mathrm{T}$ and B. clausii $\mathrm{O} / \mathrm{C}$, pure culture of $B$. clausii $\mathrm{T}$ removed single $\mathrm{CFX}$ and the coculture within 4 hours as well as the CTX removed by the pure culture within 8 hours. Hence, there was a faster removal rate of CTX and CFX compared to other treatments. Expression levels of relevant genes during the degradation of antibiotics. It was seen the degradation of CFX and CPR was associated to the overexpression of the bcl1 gene. No correlation took place between the overexpression of bcll and removal efficiency of antibiotics. Biological toxicity of degradation products. The result showed a reduction in the biotoxicity of CFX and CPR, when looking into their degradation products. ${ }^{19}$

Investigation of maternal exposure to cefuroxime before or after giving birth influences the gut microbiota in infants

Many countries have women undergoing cesarean delivery than vaginal delivery. Cesarean delivery shows a higher rate of urinary tract infection, wound infection and endometritis following child birth. Mothers are usually given prophylactic administration of antibiotics to lower the threat of the surgical site infection. Cefuroxime (1500 $\mathrm{mg}$ ) is administered by the mother before the skin incision of after the umbilical cord clamping. This study involved 42 pregnant women for chosen cesarean delivery at a hospital in Denmark. They were assigned randomly to receive cefuroxime before a skin cut or after the cut if the umbilical cord. Infants' fecal samples were collected at the age of 10 days and 9 months. A 16 S ribosomal RNA gene amplicon high-throughput sequencing was used to find the composite of the gut microbiome. Before the antimicrobial susceptibility test was carried out by disk diffusion, grampositive cocci and Enterobacteriaceae was isolated and identified. The results showed no strong difference in the composition of the gut microbiome between the infants whose mother had cefuroxime either before or after the cesarean delivery, though at 9 months of age the observed species was huge in infants whose mothers received cefuroxime after the cord was clamped rather than at 10 days of age. On the 10th day, there was no difference seen in antimicrobial susceptibility of Staphylococcus spp, Enterobacteriaceae and Enterococcus spp. There is no major effect in infants on their gut microbiota and bacterial antibiotic resistance traits no matter the timing of cefuroxime when given to mothers undergoing cesarean delivery. Fecal samples (86) were gotten from 44 babies in 10 days (pre, $\mathrm{N}=24$; post $\mathrm{N}=20$ ) and 9 months (pre, $\mathrm{N}=22$; post, $\mathrm{N}=20$ ) with 2 twins set of babies contributing at 10 days as well as a set of twin babies at 9 months of age. On the 10th day, no variance was seen in alpha-diversity events between the groups by the observed species (pre $=148 \pm 49$, post $=151 \pm 59, \mathrm{p}=0.76$ as well as Shannon diversity pre $=3.7 \pm 1$, post $=4.0 \pm 1, \mathrm{p}=0.60$ ). There was a raise seen on the number of observed species at 9 months of age in the post group (pre $=361 \pm 141$, post $=496 \pm 151$, $\mathrm{p}=0.012$ and the Shannon diversity index did not vary between the groups (pre $=5.2 \pm 1.1$, post $=6.0 \pm 1.3, \mathrm{p}=0.062$ ). The distance metrics showed no influence on the gut microbiota composition via the umbilical cord at an early life antibiotic contact as well diet had no effect after the 10th day. No clear difference was seen in the antibiotic susceptibility between the isolates pre and post group infants with only pre and post gram negative isolates being resistant to either 1 of the 3 tested antibiotics. Resistance against ampicillin was distributed amongst the gramnegative isolates from the pre and post group isolates. Prophylactic antibiotics are administered by mothers undergoing cesarean delivery to stop post-surgery complications. This process involved a single dose of cefuroxime after umbilical cord clamping to elude placental movement of the antibiotic to the fetus. Ten days was selected for an early time point as the gut microbiota will start to remain immediately after birth and nine months was for a long-term time point to represent the time after an outline of the weaning food and the slow transition towards an adult-like gut microbiota. This study researched the effect of very early-life contact to cefuroxime on gut microbiota composition. The maternal milk microbiome can be influenced by maternal exposure to cefuroxime near the time of birth. ${ }^{20}$

The gut microbiome and the immune system as a result of antibiotic therapy when effected by a mare's milk prebiotic supplementation

Antibiotics have been known to have effects for a long time on the human microbiome. Many kinds of diseases such as antibiotic-related diarrhea, bowel disease (inflammatory), obesity and various other health issues occur after longterm modifications in the gut microbiome. Supplements like probiotic and prebiotic are recommended regulate the function and structure of the human microbiome. The aim of the study was to know how a mare's milk prebiotics can impact the range of the gut bacterial population as well as the local immune system when managed through and following a course of antibiotic. Detected with bilateral bronchopneumonia, six children aged 4-5 years were prescribed with an antibiotic called cefuroxime (cephalosporin). Three children ingested the mare's milk prebiotics out of the other three that did not during the 60 days of the study. During this antibiotic therapy fecal samples were collected every day as well as every five days after the antibiotic treatment concluded. The total DNA was isolated including the taxonomic structure of the gut microbiome examined medically and in detail by 
sequencing the $16 \mathrm{~S}$ rRNA gene (V1-V3 region). Evaluation of the local immune status was carried out by the MULTIPLEX MAP platform. The last day of the study showed the relative abundance of 11 genera reduced and did not recover including the abundance of Bacteroides did not alter in either group as well. There was an increase in numbers from Abiotrophia, Christensenella, Anaerotruncus, Rothia, Turicibacter, Acinetobacter and Holdemani on the fifth day and remained constant during the period of the study. The study showed a decrease in the level of pro-inflammatory and anti-inflammatory chemokines (cytokines). ${ }^{21}$

\section{L. plantarum strains isolated from Katak for antibiotic susceptibility}

Many Lactobacillus species have been acknowledged as microbes with qualified presumption of safety (QPS) in the EFSA's list. L. plantarum is one of the widely species with an evidence of probiotic potential and scientific significance. Before implementation, every strain should complete many requirements. One of the EFSA's vital standards concerning the safety of probiotics is antibiotic susceptibility to avoid the antibiotic resistance gene to transfer to any opportunistic pathogens in the gut. The study assessed $14 \mathrm{~L}$. plantarum for susceptibility to 21 antibiotics from various groups. Resistant strains were seen at a higher number towards 12 antibiotics such as aminoglycosides-gentamicin, penicillins-penicillin, macrolides-clarithromycin, piperacillin, tetracyclinestetracycline, IIIth generation cephalosporins-cefotaxime, ceftazidime, ceftriaxone, quinolones-nalidixic acid, levofloxacin, ciprofloxacin, glycopeptides-vancomycin. The other tested antibiotics showed strain-specific antibiotic-sensitivity patterns. Probiotic strains were considered as an advantage, only when it is not transferable. Bearing in mind the usage of the final as agents accompanying antibiotic therapy, the susceptibility patterns of a few confirmed candidate probiotic is also vital. The 14 tested strains were obtained from strainspecific antibiotic susceptibility. These antibiotic susceptibility patterns differed between the confirmed lactobacilli and antibiotics. Antibiotic susceptibility of lactobacilli to inhibitors of cell wall synthesis. All 14 tested strains of beta-lactam antibiotics depicted resistance towards penicillin as well resistance to piperacillin was hugely widespread (10 out 14 strains). L. plantarum isolates were found susceptible to meropenem, ampicillin and amoxicillin. Antibiotic susceptibility of Lactobacilli to inhibitors of protein synthesis resistant strains were seen against the aminoglycoside gentamicin. Between 70-92\% of confirmed strains were seen to be susceptible to clarithromycin, chloramphenicol, doxycycline and erythromycin. Antibiotic susceptibility of Lactobacilli to inhibitors of the nucleic synthesis First, second and third generation quinolones like nalidixic acid, levofloxacin and ciprofloxacin had high resistance detected. Rifampin was found to be resistant towards four strains. Each candidate probiotic strain had to be evaluated first in vitro. Fourteen Lactobacillus strains were isolated from Katak. The
Lactobacilli originated from one sample of Katak and was observed diversity in the range of antibiotic susceptibility was unforeseen. Antibiotics have some limitations concerning their spectrum of antibacterial activity in human/animal. Many studies have exposed penicillin's resistance in $L$. plantarum strain from various habitats as well as penicillin resistance has been seen in starter lactic acid bacteria and probiotic. There is no evidence on Lactobacillus signifying the transfer of resistance genes for these $\beta$ - lactam antibiotics. ${ }^{22}$

\section{CONCLUSION}

This research review's purpose was to help the reader understand different aspects posed by the research on the therapeutic enhancements of antibiotics with probiotics. This was significant because it gives insights about usage of probiotics with antibiotics. There has been much research and discussion conducted on these opinions of usage and enhancements of both. Most of the research found, shows the enhancement and combined usage of both. More research and testing is required to gain a better understanding of the topic.

Funding: No funding sources Conflict of interest: None declared Ethical approval: Not required

\section{REFERENCES}

1. Sharma JB, Chanana C, Kumar S, Roy K, Malhotra N. Comparison of ofloxacin \& ornidazole with probiotic versus doxycycline \& metronidazole for the outpatient treatment of pelvic inflammatory disease. J K Sci. 2007;9(2).

2. Ashraf R, Shah NP. Antibiotic resistance of probiotic organisms and safety of probiotic dairy products. Int Food Res J. 2011;18(3):837-53.

3. Monk JP, Campoli-Richards DM. Ofloxacin a review of its antibacterial activity, pharmacokinetic properties and therapeutic use. Drugs. 1987;33(4):346-91.

4. Tripathy S, Adhya A. Ofloxacin induced leucopenia in complicated falciparum malaria: a case report. Cases J. 2009;2:7097.

5. Hayakawa I, Atarashi S, Yokohama S, Imamura M, Sakano K, Furukawa M. Synthesis and antibacterial activities of optically active ofloxacin. Antimicrob Agents Chemother. 1986;29(1):163-4.

6. Ishibashi N, Yamazaki S. Preobiotics and safety. Am J Clin Nutr. 2001;73(2):465-70.

7. Monk JP, Campoli-Richards DM. Ofloxacin a review of its antibacterial activity, pharmacokinetic properties and therapeutic use. Drugs. 1987;33(4):346-91.

8. Williams NT. No Title. Am J Heal Pharm. 2010; Oxford Uni:Probiotics.

9. Boyanova L, Mitov I. Coadministration of probiotics with antibiotics: why, when and for how long? Expert Rev Anti Infect Ther. 2012;10(4):407-9.

10. Tolone S, Pellino V, Vitaliti G, Lanzafame A, Tolone C. Evaluation of Helicobacter Pylori eradication in 
pediatric patients by triple therapy plus lactoferrin and probiotics compared to triple therapy alone. Ital $\mathbf{J}$ Pediatr. 2012;38(1):63.

11. Stillits HV, Andrade A, Matos C, Lopes J, Pires T, Joaquim JJ. Preventing antibiotic-associated diarrhea. J Spec Pediatr Nurs. 2010;15(2):160-2.

12. Li B, Zheng J, Zhang $X$, Hong S. Probiotic Lactobacillus shirota improves efficacy of amoxicillin sulbactam against childhood fast breathing pneumonia in a randomized placebo controlled double blind clinical study. J Clin Biochem Nutr. 2018;63(3):2337.

13. Influence of probiotic mix.

14. Gong Y, Li Y, Sun Q. Probiotics improve efficacy and tolerability of triple therapy to eradicate Helicobacter pylori: a meta-analysis of randomized controlled trials Int J Clin Exp Med. 2015;8(4):6530-43.

15. Kabbani TA, Pallav K, Dowd SE, Villafuerte-Galvez J, Vanga RR, Castillo NE, et al. Prospective randomized controlled study on the effects of Saccharomyces boulardii CNCM I-745 and amoxicillin-clavulanate or the combination on the gut microbiota of healthy volunteers. Gut Microbes. 2017;8(1):17-32.

16. Shafaghi A, Pourkazemi A, Khosravani M, Asl SF, Maafi AA, Roshan ZA, et al. The effect of probiotic plus prebiotic supplementation on the tolerance and efficacy of Helicobacter pylori eradication quadruple therapy: a randomized prospective double blind controlled trial. Middle East J Dig Dis. 2016;8(3):17988.

17. Ladirat SE, Schoterman MHC, Rahaoui H, Mars M, Schuren FHJ, Gruppen H, et al. Exploring the effects of galacto-oligosaccharides on the gut microbiota of healthy adults receiving amoxicillin treatment. Br J Nutr. 2014;112(4):536-46.
18. Du YQ, Su T, Fan JG, Lu YX, Zheng P, Li XH, et al. Adjuvant probiotics improve the eradication effect of triple therapy for Helicobacter pylori infection. World J Gastroenterol. 2012;18(43):6302-7.

19. Govender M, Choonara YE, Vuuren VS, Kumar P, Du TLC, Pillay V. Design and evaluation of an oral multiparticulate system for dual delivery of amoxicillin and Lactobacillus acidophilus. Future Microbiol. 2016;11(9):1133-45.

20. Dobreva L, Koprinarova N, Bratchkova A, Danva S. Antibiotic susceptibility of lactobacillus plantarum strains, isolated from Katak. Bulg J Vet Med. 2020.

21. Kong XX, Jiang JL, Qiao B, Liu H, Cheng JS, Yuan YJ. The biodegradation of cefuroxime, cefotaxime and cefpirome by the synthetic consortium with probiotic Bacillus clausii and investigation of their potential biodegradation pathways. Sci Total Environ. 2019;651:271-80.

22. Kamal SS, Hyldig N, Krych Ł, Greisen G, Krogfelt $\mathrm{KA}$, Zachariassen $\mathrm{G}$, et al. Impact of early exposure to cefuroxime on the composition of the gut microbiota in infants following cesarean delivery. J Pediatr. 2019;210:105.

23. Nurgaziyev M, Aitenov $\mathrm{Y}$, Khassenbekova Z, Akpanova S, Rysbekov K, Kozhakhmetov S, et al. Effect of Mare's milk prebiotic supplementation on the gut microbiome and the immune system following antibiotic therapy. Biodiversitas. 2020;21(11).

Cite this article as: Kwatra B, Raghavendra R, Patre RS, Kuntawala DH. Therapeutic enhancement of antibiotics using probiotics: a review. Int J Basic Clin Pharmacol 2021;10:1025-37. 